$11-1-2012$

\title{
Single Sampling Plans for Variables Indexed by AQL and AOQL with Measurement Error
}

\section{R. Sankle}

Vikram University, India, Ujjain (M. P.)

J.R. Singh

Vikram University, India, Ujjain (M. P.)

Follow this and additional works at: http://digitalcommons.wayne.edu/jmasm

Part of the Applied Statistics Commons, Social and Behavioral Sciences Commons, and the Statistical Theory Commons

\section{Recommended Citation}

Sankle, R. and Singh, J.R. (2012) "Single Sampling Plans for Variables Indexed by AQL and AOQL with Measurement Error," Journal of Modern Applied Statistical Methods: Vol. 11 : Iss. 2 , Article 12.

DOI: $10.22237 /$ jmasm/1351743060

Available at: http://digitalcommons.wayne.edu/jmasm/vol11/iss2/12

This Regular Article is brought to you for free and open access by the Open Access Journals at DigitalCommons@WayneState. It has been accepted for inclusion in Journal of Modern Applied Statistical Methods by an authorized editor of DigitalCommons@WayneState. 


\title{
Single Sampling Plans for Variables Indexed by AQL and AOQL with Measurement Error
}

\author{
R. Sankle J. R. Singh \\ Vikram University, \\ India, Ujjain (M. P.)
}

Single sampling plans are investigated for variables indexed by acceptable quality level (AQL) and average outgoing quality limit (AOQL) under measurement error. Procedures and tables are provided for selection of single sampling plans for variables for given AQL and AOQL when rejected lots are 100\% inspected for replacement of a nonconforming unit. For a particular sampling plan in operation for an observed measurement, a method for determining true operating characteristic (OC) functions and average outgoing quality (AOQ) is described for various error sizes.

Key words: Measurement error, AQL, AOQL.

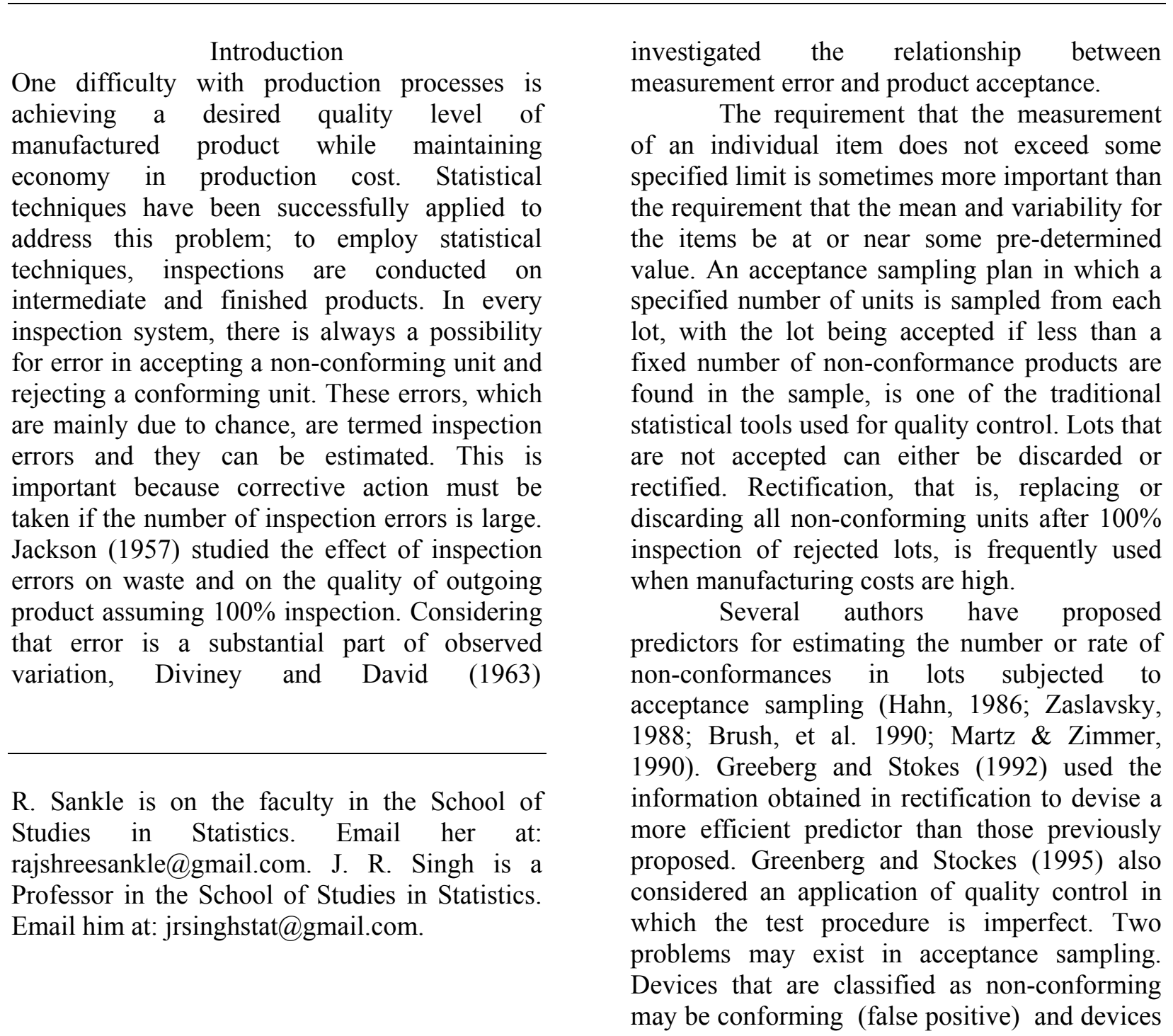




\section{R. SANKLE \& J. R. SINGH}

that are classified as conforming may be nonconforming (false negative). Johnson, et al. (1991) provided expressions and tables of the average outgoing quality for many types of sampling plans when the false positive and false negative rates are known. Lindsay (1985) described methods for estimating the probability of false positives and false negatives and the rates and numbers of non-conformances when a sample is repeatedly inspected. However, these authors do not consider plans with rectification.

A lot-by-lot rectification inspection scheme for a series of lots calls for $100 \%$ inspection of rejected lots under the application of a sampling plan. If it is preferable to use a single sampling plan for variables under a rectification inspection scheme, the index for the selection of the sampling plan will be the average outgoing quality limit (AOQL), which is the worst average quality the consumer will receive in the long run, regardless of the incoming quality. Rejected lots are often a nuisance to the producers because they result in extra work and extra cost. If too many lots are rejected the reputation of the producer or supplier may be damaged. From the producer's point of view, it is preferable to fix an acceptable quality level (AQL) by designing a sampling plan such that, if the incoming product quality is maintained at AQL most of the lots, for example $95 \%$, will be accepted during the sampling inspection stage. Thus, designing sampling inspection plans indexed by AQL and AOQL satisfies both the producer and consumer whenever rectifying inspection is necessary. The predictors are generally assumed to be measured without error, but this is often not the case.

To identify the parameter in the model, the following assumptions are made concerning measurement errors. First, it is assumed that the true values and the measurement errors are uncorrelated and that the mean of the measurement errors is zero. Second, the measurement errors are assumed to be normally distributed with zero mean and a constant known variance. Third, the true values are assumed to be normally distributed with a mean estimated by the mean of the observed values and a variance estimated using the reliability of the observed values. The reliability of a variable measured with error is the ratio of the variance of the true values to the variance of the observed values; the closer this ratio is to 1 the more reliable the measurement. The reliability can be provided by reliability coefficients (Hand, 2004). Alternatively, a range of plausible reliabilities can be explored to carry out a sensitivity analysis of the results to estimate the severity of the unobserved measurement error.

This study examines single sampling plans for variables indexed by AQL and AOQL under measurement error. Procedures and tables are provided for selecting single sampling plans for variables for given AQL and AOQL when rejected lots are $100 \%$ inspected for replacement of nonconforming units. For a particular sampling plan in operation for observed measurement, a method of determining the OC function and AOQ curves is described for various errors sizes.

Model description for Variable Single Sampling Plan indexed by AQL and AOQL under Measurement Errors:

Consider the distribution of the true quality characteristics $\mathrm{x}$ to be normal with mean $\mu$ and known standard deviation $\sigma_{p}$. The density function is:

$$
\mathrm{f}(\mathrm{x})=\frac{1}{\sigma_{p}} \Phi\left(\frac{\mathrm{x}-\mu}{\sigma_{\mathrm{p}}}\right),
$$

where $\Phi(\mathrm{x})$ is the standardized normal probability density function given by

$$
\Phi(x)=\frac{1}{\sqrt{2 \pi}} e^{-\frac{1}{2} x^{2}}
$$

The mean and standard deviation of the observed measurement $(\mathrm{X}=\mathrm{x}+\mathrm{e})$ can be written as

$$
\mathrm{E}(\mathrm{X})=\mathrm{E}(\mathrm{x})+\mathrm{E}(\mathrm{e})=\mu
$$

where $\mu$ is the mean of $x$ and $e$ is the random error at measurement and is independent of $\mathrm{x}$, and

$$
\mathrm{V}(\mathrm{X})=\mathrm{V}(\mathrm{x})+\mathrm{V}(\mathrm{e})=\sigma_{\mathrm{p}}^{2}+\sigma_{\mathrm{e}}^{2}=\sigma_{\mathrm{X}}^{2}
$$


The correlation coefficient $\rho$ between the true and observed measurement is given by

$$
\begin{aligned}
\rho & =\frac{E\{(x-\mu)(X-\mu)\}}{\sigma_{p} \sigma_{X}} \\
& =\frac{E\left\{(x-\mu)^{2}+(x-\mu) e\right\}}{\sigma_{p} \sigma_{X}} .
\end{aligned}
$$

Noting that $\mathrm{x}$ and $\mathrm{e}$ are independent, $\mathrm{E}(\mathrm{e})=0$ and $\mathrm{E}(\mathrm{x})=\mu$, it can be shown that

$$
\begin{gathered}
\rho=\frac{\sigma_{p}{ }^{2}}{\sigma_{p} \sigma_{X}} \\
=\frac{\sigma_{p}}{\sigma_{X}} .
\end{gathered}
$$

The relation between the size of measurement error $r$ and correlation coefficient $\rho$ is:

$$
\rho=\frac{r}{\sqrt{1+r^{2}}}
$$

where

$$
r=\frac{\sigma_{p}}{\sigma_{e}}
$$

In referencing a single sampling variable plan when $\sigma_{p}$ is known, the following symbols are used:

L: Lower specification limit;

U: Upper specification limit;

N: Sample size;

$k$ : Acceptance parameter; and

$\bar{x}$ : Sample mean

$$
\Phi(y)=\int_{-\infty}^{y}\left(\frac{1}{\sqrt{2 \pi}}\right) \exp \left(-\frac{1}{2} z^{2}\right) d z,
$$

where $z \sim N(0,1)$.

The acceptance criterion for the single sampling plan is: For the upper specification limit, accept the lot if,

$$
\bar{x}+k \sigma_{P} \leq U,
$$

and, for the lower specification limit, accept the lot if

$$
\bar{x}-k \sigma_{P} \geq L
$$

The fraction nonconforming in a given lot is

$$
\Phi\left(-K_{p}\right)=p
$$

with

$$
K_{p}=\frac{U-\mu}{\sigma_{p}}
$$

where $K_{p}$ is the $p$ percent point of the standard normal distribution. If $p$ is the proportion defective in the lot, then

$$
U=\mu+K_{p} \sigma_{p}
$$

and its probability of acceptance under measurement error will be

$$
P_{a}(p)=\Phi(w)
$$

with

$$
w=\left(K_{p}-k\right) \frac{\sqrt{n}}{\rho} .
$$

If the quality of the accepted lot is $p$ and all nonconforming units found in the rejected lots are replaced by conforming units in a rectification inspection scheme, the AOQ can be approximated as

$$
\mathrm{AOQ}=p \cdot \mathrm{P}_{\mathrm{a}}(p)
$$

If $p_{m}$ is the proportion non-conforming at which AOQ is maximum, then

$$
\mathrm{AOQL}=p_{m} \mathrm{P}_{\mathrm{a}}\left(p_{m}\right) .
$$




\section{R. SANKLE \& J. R. SINGH}

If AQL $\left(p_{1}\right)$ is prescribed, then the corresponding value of $\mathrm{K}_{\mathrm{AQL}}$ or $\mathrm{K}_{1}$ will be fixed, and if $\mathrm{P}_{\mathrm{a}}\left(p_{1}\right)$ is fixed at $95 \%$, then, $\mathrm{w}_{\mathrm{AQL}}=\mathrm{w}_{1}=$ 1.645; hence,

$$
1.645=\left(\mathrm{K}_{1}-\mathrm{k}\right) \frac{\sqrt{\mathrm{n}}}{\rho},
$$

so that for a given AQL, $k$ is determined by the sample size $n$.

\section{Results}

Table 1 is used for selecting a single sampling variables plan under measurement errors for known $\sigma$ case. For example, if the AQL is fixed at $1 \%$, the AOQL is fixed at $1.25 \%$ and $r=2,4$, 6 and $\infty$, Table 1 yields $\mathrm{n}=39,27,26$ and 25, and $k=1.989,1.990,1.992$ and 1.998, respectively. It shows that, when the size of the error increases, the value of $n$ increases and, due to measurement errors, the sample sizes are affected but there is a very minor change in acceptance parameter $k$. Further, suppose that it is decided to use $\sigma$, an acceptance criterion where $\sigma_{\mathrm{p}}$ is known to be 2.0. Let there exist an upper specific limit $U=10.0$ and a unit for which the quality characteristic $\mathrm{x}>\mathrm{U}$ is considered as nonconforming.

Table 2 shows the performance characteristics of a sampling plan with $n=25$ and $k=2.0$ under a rectifying inspection scheme. If the true process average quality is operating at AQL $(\mu=5.346)$ and $r=\infty$, then $95 \%$ of the lots submitted will be accepted during the sampling inspection stage itself and only $5 \%$ of the rejected lots will be rectified by replacing non-conforming units with conforming units. In such a case, the AOQ will be only about $1 \%$. If the submitted quality deteriorates to $1.79 \%$ (error free case, that is, $r=\infty$ ), then only about $70 \%$ of the lots will be accepted by the sampling plan and approximately one out of every three lots will be rejected and rectified. The AOQ in such a case will not exceed the AOQL of $1.25 \%$ fixed, meaning that, irrespective of the product quality submitted by the producer, the consumer will receive an average quality not worse than $1.25 \%$ under the rectification scheme. The worst case is when $r=$ 2; the AOQ in such a case will just exceed the AOQL of $1.25 \%$ fixed for different errors sizes. The values of $\mathrm{P}_{\mathrm{a}}\left(\mathrm{p}_{\mathrm{m}}\right)$ for known $\sigma$ case are presented in Table 3, and a visual comparison of AOQ curves for different error sizes is shown in Figure 1. As Figure 1 illustrates, the effect of measurement error is serious on the AOQ curves.

When using Table 1 to select sampling plans, limitations of plans indexed by AOQL under measurement error must be taken into account. Sampling with rectification of rejected lots reduces the average percentage of nonconforming items in the lots; however, it also introduces non-homogeneity in the series of lots finally accepted. That is, any particular lot will have a quality of $\mathrm{p} \%$ or $0 \%$ non-conforming depending on whether the lot is accepted or rectified. Thus, the assumption underlying the AOQL principle is that the homogeneity in the qualities of individual lots is unimportant and only average quality matters.

Table 3 gives $\mathrm{P}_{\mathrm{a}}\left(\mathrm{p}_{\mathrm{m}}\right)$ values for the plans given in Table 1. If $\mathrm{AQL}$ is $0.25 \%$, AOQL is $1.25 \%$ and $\mathrm{r}=2,4,6$ and $\infty$, then $\mathrm{P}_{\mathrm{a}}\left(\mathrm{p}_{\mathrm{m}}\right)$ is $0.354,0.342,0.340$ and 0.338 , respectively, and $\mathrm{p}_{\mathrm{m}}=\mathrm{AOQL} / \mathrm{P}_{\mathrm{a}}\left(\mathrm{p}_{\mathrm{m}}\right)$ for $\mathrm{r}=2,4,6$ and $\infty$, is $3.53 \%, 3.65 \%, 3.67 \%, 3.69 \%$. Thus, if the lot quality is $3.69 \%$ then, on average, among every three lots passed on to the consumer two will be free from non-conforming items while the third lot will contain $3.69 \%$ non-conforming items: this is about 15 times the AQL specified. In order to avoid such error, the producer should maintain the process quality approximately at the set AQL because a high rate of rejecting lots at $p=p_{m}$ will also indirectly put pressure on the producer to improve the submitted quality.

\section{References}

Brush, G. G., Hoadley, B., \& Saperstein B. (1990). Estimating outgoing quality using the quality measurement plan. Technometrics, 32, 31-41.

Diviney, T. E., \& David, N. A. (1963). A note on the relationship between measurement error and product acceptance. Journal of Industrial Engineering, 14, 218-219. 
SAMPLING PLANS FOR AQL AND AOQL VARIABLES WITH MEASUREMENT ERROR

Table 1: Single Sampling Plans for Variables Indexed by AQL and AOQL under Measurement Error

\begin{tabular}{|c|c|c|c|c|c|c|c|c|c|c|c|c|c|}
\hline \multirow{2}{*}{$\mathrm{r}$} & \multirow{2}{*}{$\begin{array}{c}\text { AOQL } \\
(\%)\end{array}$} & \multicolumn{12}{|c|}{ AQL (\%) } \\
\hline & & 0.040 & 0.065 & 0.100 & 0.150 & 0.250 & 0.400 & 0.650 & 1.000 & 1.500 & 2.500 & 4.000 & 6.500 \\
\hline \multirow{17}{*}{2} & 0.040 & $\begin{array}{c}74, \\
3.107\end{array}$ & & & & & & & & & & & \\
\hline & 0.080 & $\begin{array}{c}19 \\
2.905\end{array}$ & $\begin{array}{c}75 \\
2.971\end{array}$ & & & & & & & & & & \\
\hline & 0.125 & $\begin{array}{c}10 \\
2.753\end{array}$ & $\begin{array}{c}19 \\
2.765\end{array}$ & $\begin{array}{c}63 \\
2.826\end{array}$ & & & & & & & & & \\
\hline & & 7 & 10 & 17 & 43, & & & & & & & & \\
\hline & 0.200 & 2.604 & 2.595 & 2.606 & 2.654 & & & & & & & & \\
\hline & 0.320 & $\begin{array}{c}5 \\
2458\end{array}$ & $\begin{array}{c}6 \\
2438\end{array}$ & $\begin{array}{c}9 \\
2429\end{array}$ & $\begin{array}{c}14 \\
2437\end{array}$ & $\begin{array}{c}47 \\
2504\end{array}$ & & & & & & & \\
\hline & & 3 & 4 & 5 & 8 & 14 & 49 & & & & & & \\
\hline & 0.500 & 2.317 & 2.289 & 2.271 & 2.263 & 2.276 & 2.350 & & & & & & \\
\hline & 0.800 & 3 & 3 , & 4, & 5 & 7, & 12 & 47 & & & & & \\
\hline & & & 2.235 & 2.104 & 2.083 & & & 2.174 & & & & & \\
\hline & 1.250 & & $\begin{array}{l}2 \\
1965\end{array}$ & $\begin{array}{l}3, \\
1937\end{array}$ & $\begin{array}{c}3, \\
1914\end{array}$ & $\begin{array}{l}4, \\
1894\end{array}$ & $\begin{array}{c}6 \\
1.888\end{array}$ & 12 & $\begin{array}{c}39 \\
1989\end{array}$ & & & & \\
\hline & & & & 2 & 2 & 3 & 4 & 6 & 10 & 25 & & & \\
\hline & 2.000 & & & 1.748 & 1.722 & 1.694 & 1.677 & 1.672 & 1.692 & 1.760 & & & \\
\hline & 3.200 & & & & & $\begin{array}{c}2 \\
477\end{array}$ & $\begin{array}{l}3, \\
454\end{array}$ & $\begin{array}{c}2, \\
438\end{array}$ & $\begin{array}{c}5 \\
436\end{array}$ & $\begin{array}{c}7 \\
1453\end{array}$ & $\begin{array}{c}26 \\
1549\end{array}$ & & \\
\hline & & & & & & & & 2 & 3 & 4 & 7 & & \\
\hline & 5.000 & & & & & & & 1.143 & 1.186 & 1.186 & 1.214 & 1.326 & \\
\hline & 8.000 & & & & & & & & $\begin{array}{c}2 \\
0.889\end{array}$ & $\begin{array}{c}2, \\
0.882\end{array}$ & $\begin{array}{c}3, \\
0.887\end{array}$ & $\begin{array}{c}6, \\
0.923\end{array}$ & $\begin{array}{c}21 \\
1.058\end{array}$ \\
\hline \multirow{19}{*}{4} & 0.040 & $\begin{array}{c}52, \\
3.109\end{array}$ & & & & & & & & & & & \\
\hline & 0.080 & $\begin{array}{c}17 \\
2.936\end{array}$ & $\begin{array}{c}51 \\
2.969\end{array}$ & & & & & & & & & & \\
\hline & 0125 & 10 & 17 & 45 , & & & & & & & & & \\
\hline & 0.120 & 2.800 & 2.795 & 2.828 & & & & & & & & & \\
\hline & 0.200 & $\begin{array}{c}6 \\
2665\end{array}$ & $\begin{array}{c}9 \\
2643\end{array}$ & $\begin{array}{c}15 \\
2639\end{array}$ & $\begin{array}{c}33 \\
2662\end{array}$ & & & & & & & & \\
\hline & & 4 & 6 & 8 & 12 & 34 & & & & & & & \\
\hline & 0.320 & 2.532 & 2.500 & 2.481 & 2.475 & 2.508 & & & & & & & \\
\hline & 0500 & 3 , & 4 , & 5 , & 7 , & 12 & 34, & & & & & & \\
\hline & 0.500 & 2.402 & 2.365 & 2.337 & 2.318 & 2.312 & 2.351 & & & & & & \\
\hline & 0.800 & 2 & 3, & 4 & 4, & 7 & 11 & 32 & & & & & \\
\hline & & & 2.217 & 2.184 & 2.157 & 2.133 & 2.130 & 2.175 & & & & & \\
\hline & 1.250 & & $\begin{array}{c}2, \\
2067\end{array}$ & 3, & $\begin{array}{c}3, \\
2000\end{array}$ & $\begin{array}{c}4, \\
1967\end{array}$ & $\begin{array}{c}6 \\
1946\end{array}$ & 10, & $\begin{array}{c}27, \\
1990\end{array}$ & & & & \\
\hline & & & & 2 & 2 & 3 & 4 & 5 & 9 & 19, & & & \\
\hline & 2.000 & & & 1.857 & 1.823 & 1.784 & 1.754 & 1.734 & 1.734 & 1.770 & & & \\
\hline & 3.200 & & & & & 2 & 2 & 3 & 4 & 7 & 19 & & \\
\hline & & & & & & 1.585 & 1.550 & 1.521 & 1.504 & 1.503 & 1.555 & & \\
\hline & 5.000 & & & & & & & $\begin{array}{c}2, \\
299\end{array}$ & $\begin{array}{c}3, \\
1275\end{array}$ & $\begin{array}{c}4, \\
1260\end{array}$ & $\begin{array}{c}6 \\
1264\end{array}$ & 17 & \\
\hline & 8 & & & & & & & & 2 & 2 & 3 & 5 & 15 \\
\hline & 8.000 & & & & & & & & 1.003 & 0.982 & 0.968 & 0.977 & 1.055 \\
\hline
\end{tabular}




\section{R. SANKLE \& J. R. SINGH}

Table 1 (continued): Single Sampling Plans for Variables Indexed by AQL and AOQL under Measurement Error

\begin{tabular}{|c|c|c|c|c|c|c|c|c|c|c|c|c|c|}
\hline \multirow{2}{*}{$\mathrm{r}$} & \multirow{2}{*}{$\begin{array}{c}\text { AOQL } \\
(\%)\end{array}$} & \multicolumn{12}{|c|}{ AQL (\%) } \\
\hline & & 0.040 & 0.065 & 0.100 & 0.150 & 0.250 & 0.400 & 0.650 & 1.000 & 1.500 & 2.500 & 4.000 & 6.500 \\
\hline \multirow{20}{*}{6} & 0.040 & 49 & & & & & & & & & & & \\
\hline & & $\begin{array}{c}3.111 \\
17\end{array}$ & & & & & & & & & & & \\
\hline & 0.080 & 2.942 & 2.971 & & & & & & & & & & \\
\hline & 0.125 & $\begin{array}{c}10 \\
2809\end{array}$ & $\begin{array}{c}16 \\
2802\end{array}$ & $\begin{array}{c}42, \\
2829\end{array}$ & & & & & & & & & \\
\hline & & 6 & 9 & 14 & 32 , & & & & & & & & \\
\hline & 0.200 & 2.677 & 2.653 & 2.646 & 2.666 & & & & & & & & \\
\hline & 0.320 & 4 & 6 & 8 & 12 & 33 & & & & & & & \\
\hline & & 2.546 & 2.513 & 2.491 & 2.483 & 2.511 & & & & & & & \\
\hline & 0.500 & $\begin{array}{c}3, \\
2419\end{array}$ & $\begin{array}{c}4, \\
2380\end{array}$ & $\begin{array}{c}5, \\
2350\end{array}$ & $\begin{array}{c}7 \\
2329\end{array}$ & $\begin{array}{c}12, \\
2320\end{array}$ & $\begin{array}{c}32, \\
2354\end{array}$ & & & & & & \\
\hline & & 2 & 3 & 4 & 4 & $\begin{array}{c}2.520 \\
6\end{array}$ & 11 & & & & & & \\
\hline & 0.800 & 2.277 & 2.235 & 2.199 & 2.171 & 2.145 & 2.138 & 2.174 & & & & & \\
\hline & 1250 & & 2 & 3 & 3 & 4, & 6 & 10, & 26, & & & & \\
\hline & 1.250 & & 2.088 & 2.050 & 2.017 & 1.981 & 1.958 & 1.954 & 1.992 & & & & \\
\hline & 2.000 & & & 2 & 2 & 3 & 4, & 5 & 8 & 18 & & & \\
\hline & & & & & & 1.802 & 1.770 & 1.747 & 1.744 & 1.773 & & & \\
\hline & 3.200 & & & & & $\begin{array}{c}2, \\
1.606\end{array}$ & $\begin{array}{c}2, \\
1.569\end{array}$ & $\begin{array}{c}3 \\
1.537\end{array}$ & $\begin{array}{c}4 \\
1.518\end{array}$ & $\begin{array}{c}7, \\
1514\end{array}$ & $\begin{array}{l}18, \\
1558\end{array}$ & & \\
\hline & & & & & & & & 2 & 3 & 4 & 6 & & \\
\hline & 5.000 & & & & & & & 1.320 & 1.293 & 1.276 & 1.275 & 1.329 & \\
\hline & 8.000 & & & & & & & & 2 & 2 & 3 & 5 & 14 \\
\hline & & & & & & & & & & & & & \\
\hline \multirow{20}{*}{$\infty$} & 0.040 & $\begin{array}{c}47, \\
3.084\end{array}$ & & & & & & & & & & & \\
\hline & 0.080 & $\begin{array}{c}17, \\
2.900\end{array}$ & $\begin{array}{c}46 \\
2.972\end{array}$ & & & & & & & & & & \\
\hline & 0125 & 9 & 16 & 40, & & & & & & & & & \\
\hline & 0.125 & 2.754 & 2.807 & 2.831 & & & & & & & & & \\
\hline & 0.200 & 6 & 9, & 14 & 30 & & & & & & & & \\
\hline & & 2.608 & 2.661 & 2.652 & 2.670 & & & & & & & & \\
\hline & 0.320 & 4, & 6 & 8 & 12 & 31, & & & & & & & \\
\hline & & 2.464 & 2.523 & 2.500 & 2.489 & 2.513 & & & & & & & \\
\hline & 0.500 & 3, & 4, & 5 & 7, & 12 & 31, & & & & & & \\
\hline & & 2.324 & 2.392 & 2.361 & 2.338 & 2.327 & 2.355 & & & & & & \\
\hline & 0.800 & 2, & 3, & 4, & 4, & 6 & 11, & 29 & & & & & \\
\hline & & & 2.249 & 2.213 & 2.183 & 2.154 & 2.145 & 2.178 & & & & & \\
\hline & 1.250 & & 2, & 3, & 3 & 4 & 6 & 10 & 25 & & & & \\
\hline & & & 2.104 & 2.065 & 2.031 & 1.994 & 1.968 & 1.961 & 1.994 & & & & \\
\hline & 2.000 & & & 2 & 2 & 3 & $\begin{array}{c}4, \\
402\end{array}$ & 5 & 8 & 18 & & & \\
\hline & & & & & & $\begin{array}{c}1.817 \\
2\end{array}$ & $\begin{array}{c}1.783 \\
2\end{array}$ & $\begin{array}{c}1.748 \\
3\end{array}$ & $\begin{array}{c}1.741 \\
4\end{array}$ & $\begin{array}{c}1.788 \\
6\end{array}$ & & & \\
\hline & 3.200 & & & & & $\begin{array}{l}2, \\
1.623\end{array}$ & 1.585 & $\begin{array}{c}3, \\
1.551\end{array}$ & $\begin{array}{c}4, \\
1.529\end{array}$ & $\begin{array}{c}0, \\
1.522\end{array}$ & $\begin{array}{l}1 /, \\
1.561\end{array}$ & & \\
\hline & 5000 & & & & & & & 2 & 3 & 3 & 6 & 15 & \\
\hline & 3.000 & & & & & & & 1.337 & 1.308 & 1.288 & 1.284 & 1.332 & \\
\hline & 8.000 & & & & & & & & $\begin{array}{l}2, \\
1044\end{array}$ & 2, & $\begin{array}{l}3, \\
1098\end{array}$ & $\begin{array}{c}5, \\
0909\end{array}$ & 13 \\
\hline
\end{tabular}


Table 2: Performance Characteristics of the Variables Plan under Measurement Error for $\mathrm{AQL}=0.01, \mathrm{AOQL}=0.0125, \mathrm{U}=10, \mathrm{SD}=2$

\begin{tabular}{|c|c|c|c|c|c|c|}
\hline $\mathrm{r}$ & $\mu$ & $v^{\prime}$ & $\mathrm{p}(\%)$ & w & $\mathrm{Pa}$ & AOQ \\
\hline \multirow{6}{*}{2} & 5.4330 & 2.2835 & 1.12 & 1.6450 & 0.9500 & 1.0640 \\
\hline & 5.6000 & 2.2000 & 1.39 & 1.1785 & 0.8807 & 1.2245 \\
\hline & 5.8000 & 2.1000 & 1.79 & 0.6200 & 0.7324 & 1.3083 \\
\hline & 5.9000 & 2.0500 & 2.02 & 0.3407 & 0.6333 & 1.2782 \\
\hline & 6.0000 & 2.0000 & 2.28 & 0.0614 & 0.5245 & 1.1932 \\
\hline & 6.2000 & 1.9000 & 2.87 & -0.4971 & 0.3096 & 0.8889 \\
\hline \multirow{6}{*}{4} & 5.3673 & 2.3163 & 1.03 & 1.6450 & 0.9500 & 0.9757 \\
\hline & 5.4000 & 2.3000 & 1.07 & 1.5626 & 0.9409 & 1.0091 \\
\hline & 5.6000 & 2.2000 & 1.39 & 1.0586 & 0.8551 & 1.1889 \\
\hline & 5.8000 & 2.1000 & 1.79 & 0.5545 & 0.7104 & 1.2690 \\
\hline & 6.0000 & 2.0000 & 2.28 & 0.0504 & 0.5201 & 1.1832 \\
\hline & 6.2000 & 1.9000 & 2.87 & -0.4537 & 0.3250 & 0.9334 \\
\hline \multirow{6}{*}{6} & 5.3618 & 2.3191 & 1.02 & 1.6450 & 0.9500 & 0.9686 \\
\hline & 5.4000 & 2.3000 & 1.07 & 1.5491 & 0.9393 & 1.0073 \\
\hline & 5.6000 & 2.2000 & 1.39 & 1.0462 & 0.8523 & 1.1849 \\
\hline & 5.8000 & 2.1000 & 1.79 & 0.5432 & 0.7065 & 1.2621 \\
\hline & 6.0000 & 2.0000 & 2.28 & 0.0402 & 0.5160 & 1.1740 \\
\hline & 6.2000 & 1.9000 & 2.87 & -0.4627 & 0.3218 & 0.9240 \\
\hline \multirow{6}{*}{$\infty$} & 5.3460 & 2.3270 & 1.00 & 1.6450 & 0.9500 & 0.9484 \\
\hline & 5.4000 & 2.3000 & 1.07 & 1.5100 & 0.9345 & 1.0021 \\
\hline & 5.8000 & 2.1000 & 1.79 & 0.5100 & 0.6950 & 1.2415 \\
\hline & 6.0000 & 2.0000 & 2.28 & 0.0100 & 0.5040 & 1.1466 \\
\hline & 6.2000 & 1.9000 & 2.87 & -0.4900 & 0.3121 & 0.8961 \\
\hline & 6.4000 & 1.8000 & 3.59 & -0.9900 & 0.1611 & 0.5788 \\
\hline
\end{tabular}




\section{R. SANKLE \& J. R. SINGH}

Table 3: $\mathrm{Pa}(\mathrm{pm})$ Values of Known Sigma Plans Under Measurement Error

\begin{tabular}{|c|c|c|c|c|c|c|c|c|c|c|c|c|c|}
\hline \multirow{2}{*}{$\mathrm{r}$} & \multirow{2}{*}{$\begin{array}{c}\text { AOQL } \\
(\%)\end{array}$} & \multicolumn{12}{|c|}{ AQL $(\%)$} \\
\hline & & 0.040 & 0.065 & 0.100 & 0.150 & 0.250 & 0.400 & 0.650 & 1.000 & 1.500 & 2.500 & 4.000 & 6.500 \\
\hline \multirow{12}{*}{2} & 0.050 & 0.769 & & & & & & & & & & & \\
\hline & 0.080 & 0.538 & 0.774 & & & & & & & & & & \\
\hline & 0.125 & 0.413 & 0.554 & 0.781 & & & & & & & & & \\
\hline & 0.200 & 0.327 & 0.420 & 0.541 & 0.723 & & & & & & & & \\
\hline & 0.320 & 0.271 & 0.335 & 0.415 & 0.522 & 0.762 & & & & & & & \\
\hline & 0.500 & 0.235 & 0.282 & 0.338 & 0.411 & 0.546 & 0.765 & & & & & & \\
\hline & 0.800 & 0.210 & 0.245 & 0.286 & 0.336 & 0.425 & 0.549 & 0.776 & & & & & \\
\hline & 1.250 & & 0.224 & 0.255 & 0.292 & 0.354 & 0.436 & 0.567 & 0.766 & & & & \\
\hline & 2.000 & & & 0.236 & 0.263 & 0.308 & 0.364 & 0.447 & 0.558 & 0.729 & & & \\
\hline & 3.200 & & & & & 0.284 & 0.324 & 0.379 & 0.449 & 0.544 & 0.753 & & \\
\hline & 5.000 & & & & & & & 0.504 & 0.393 & 0.454 & 0.572 & 0.769 & \\
\hline & 8.000 & & & & & & & & 0.367 & 0.407 & 0.479 & 0.581 & 0.781 \\
\hline \multirow{12}{*}{4} & 0.050 & 0.723 & & & & & & & & & & & \\
\hline & 0.080 & 0.510 & 0.753 & & & & & & & & & & \\
\hline & 0.125 & 0.395 & 0.527 & 0.712 & & & & & & & & & \\
\hline & 0.200 & 0.315 & 0.402 & 0.515 & 0.723 & & & & & & & & \\
\hline & 0.320 & 0.262 & 0.323 & 0.398 & 0.498 & 0.718 & & & & & & & \\
\hline & 0.500 & 0.228 & 0.273 & 0.326 & 0.394 & 0.520 & 0.745 & & & & & & \\
\hline & 0.800 & 0.205 & 0.239 & 0.278 & 0.325 & 0.408 & 0.529 & 0.727 & & & & & \\
\hline & 1.250 & & 0.219 & 0.248 & 0.283 & 0.342 & 0.420 & 0.540 & 0.737 & & & & \\
\hline & 2.000 & & & 0.232 & 0.258 & 0.300 & 0.353 & 0.431 & 0.533 & 0.715 & & & \\
\hline & 3.200 & & & & & 0.278 & 0.316 & 0.369 & 0.434 & 0.522 & 0.718 & & \\
\hline & 5.000 & & & & & & & 0.338 & 0.383 & 0.440 & 0.548 & 0.727 & \\
\hline & 8.000 & & & & & & & & 0.361 & 0.398 & 0.465 & 0.561 & 0.750 \\
\hline
\end{tabular}


SAMPLING PLANS FOR AQL AND AOQL VARIABLES WITH MEASUREMENT ERROR

Table 3 (continued): $\mathrm{Pa}(\mathrm{pm})$ Values of Known Sigma Plans Under Measurement Error

\begin{tabular}{|c|c|c|c|c|c|c|c|c|c|c|c|c|c|}
\hline \multirow{2}{*}{$r$} & \multirow{2}{*}{$\begin{array}{c}\text { AOQL } \\
(\%)\end{array}$} & \multicolumn{12}{|c|}{ AQL (\%) } \\
\hline & & 0.040 & 0.065 & 0.100 & 0.150 & 0.250 & 0.400 & 0.650 & 1.000 & 1.500 & 2.500 & 4.000 & 6.500 \\
\hline \multirow{12}{*}{6} & 0.050 & 0.702 & & & & & & & & & & & \\
\hline & 0.080 & 0.505 & 0.728 & & & & & & & & & & \\
\hline & 0.125 & 0.391 & 0.521 & 0.711 & & & & & & & & & \\
\hline & 0.200 & 0.313 & 0.398 & 0.509 & 0.689 & & & & & & & & \\
\hline & 0.320 & 0.260 & 0.320 & 0.394 & 0.493 & 0.696 & & & & & & & \\
\hline & 0.500 & 0.227 & 0.271 & 0.324 & 0.391 & 0.514 & 0.714 & & & & & & \\
\hline & 0.800 & 0.205 & 0.238 & 0.276 & 0.323 & 0.405 & 0.519 & 0.721 & & & & & \\
\hline & 1.250 & & 0.218 & 0.247 & 0.282 & 0.340 & 0.416 & 0.535 & 0.713 & & & & \\
\hline & 2.000 & & & 0.231 & 0.256 & 0.299 & 0.351 & 0.428 & 0.528 & 0.707 & & & \\
\hline & 3.200 & & & & & 0.277 & 0.314 & 0.367 & 0.431 & 0.518 & 0.701 & & \\
\hline & 5.000 & & & & & & & 0.337 & 0.381 & 0.437 & 0.544 & 0.714 & \\
\hline & 8.000 & & & & & & & & 0.359 & 0.397 & 0.462 & 0.559 & 0.728 \\
\hline \multirow{12}{*}{$\infty$} & 0.050 & 0.700 & & & & & & & & & & & \\
\hline & 0.080 & 0.501 & 0.727 & & & & & & & & & & \\
\hline & 0.125 & 0.389 & 0.515 & 0.700 & & & & & & & & & \\
\hline & 0.200 & 0.311 & 0.395 & 0.505 & 0.663 & & & & & & & & \\
\hline & 0.320 & 0.258 & 0.318 & 0.392 & 0.489 & 0.696 & & & & & & & \\
\hline & 0.500 & 0.226 & 0.270 & 0.322 & 0.389 & 0.510 & 0.714 & & & & & & \\
\hline & 0.800 & 0.203 & 0.236 & 0.274 & 0.321 & 0.402 & 0.514 & 0.719 & & & & & \\
\hline & 1.250 & & 0.227 & 0.246 & 0.281 & 0.338 & 0.413 & 0.530 & 0.702 & & & & \\
\hline & 2.000 & & & 0.104 & 0.255 & 0.297 & 0.349 & 0.425 & 0.524 & 0.672 & & & \\
\hline & 3.200 & & & & & 0.276 & 0.313 & 0.365 & 0.428 & 0.514 & 0.696 & & \\
\hline & 5.000 & & & & & & & 0.336 & 0.379 & 0.435 & 0.540 & 0.714 & \\
\hline & 8.000 & & & & & & & & 0.358 & 0.395 & 0.460 & 0.553 & 0.720 \\
\hline
\end{tabular}




\section{R. SANKLE \& J. R. SINGH}

Figure 1: Average Outgoing Quality Curves under Measurement Error

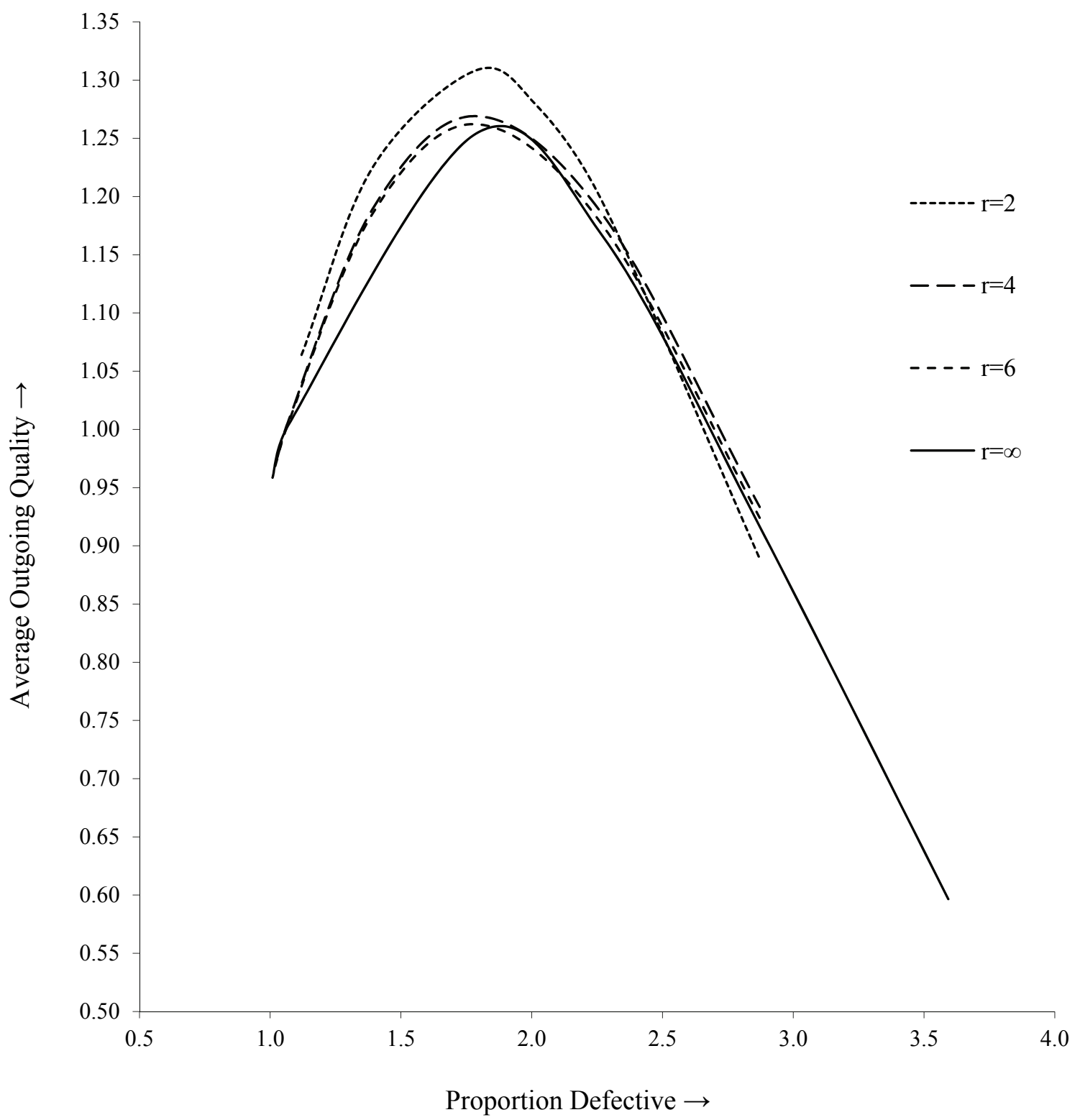

Greenberg, B. S., \& Stokes, S. L. (1992). Estimating nonconformance rates after zero defect sampling with rectification. Technometrics, 34, 203-213.

Greenberg, B. S., \& Stokes, S. L. (1995). Repetitive testing in the presence of inspection errors. Technometrics, 37, 102-111.
Hahn, G. J. (1986). Estimating the percent nonconforming in the accepted product after zero defect sampling. Journal of Quality Technology, 18, 182-188.

Hand, D. J. (2004). Measurement theory and practice. London, UK: Arnold. 
Jackson, E. J. (1957). Effect of inspection errors on waste and on quality of outgoing product. Industrial Quality Control, $\operatorname{VIX}(6), 5-8$.

Johnson, N. L., Kotz, S., \& Wu, X. (1991). Inspection errors for attributes in quality control. London, UK: Chapman \& Hall.
Martz, H. F., \& Zimmer, W.J. (1990). A nonparametric Bayes empirical Bayes procedure for estimating the percent nonconforming in accepted lots. Journal of Quality Technology, 22, 95-104.

Zaslavsky, A. (1988). Estimating nonconformity rates in C-defect sampling. Journal of Quality Technology, 20, 248-259. 